\title{
Biomasa aérea de Vochysia guatemalensis en una selva alta perennifolia de La Chinantla, Oaxaca
}

\section{Aboveground biomass of Vochysia guatemalensis in a rain tropical forest of the Chinantla, Oaxaca}

\author{
Filemón Manzano-Méndez ${ }^{1}$, Elizandro Pineda-Herrera ${ }^{2 *}$ Juan Ignacio Valdez-Hernández $^{3}$, Miguel Ángel \\ López-López ${ }^{3}$ \\ ${ }^{1}$ Asesoría e Investigación para el Manejo de Ecosistemas Forestales. CP. 71228. Oaxaca, Oaxaca \\ ${ }^{2}$ División de Ciencias Ambientales, Instituto Potosino de Investigación Científica y Tecnológica, A.C. Camino a la Presa San \\ José 2055, Col. Lomas 4 sección, CP. 78216, San Luis Potosí, San Luis Potosí, México \\ ${ }_{3}^{3}$ Postgrado Forestal, Colegio de Postgraduados Montecillo, Texcoco, Estado de México. \\ ${ }^{*}$ Autor de correspondencia: elherrera2001@yahoo.com.mx
}

Nota cientifica recibido: 26 de abril de 2016, aceptado: 04 de octubre de 2016

RESUMEN. Se estimó la biomasa aérea de Vochysia guatemalensis Donn. Smith., una especie de importancia ecológica y económica en el sur-sureste de México. Se derribaron siete árboles de cuatro categorías diamétricas (10, 15,20 y $25 \mathrm{~cm}$ ), para luego separarlos por componente estructural. De cada componente se obtuvieron muestras que se pesaron en fresco y se secaron en estufa. Con estos datos se estableció el peso seco para estimar la biomasa por componente y árbol total en cada categoría, ajustando ecuaciones alométricas mediante técnicas de regresión no lineal. Los valores de biomasa aérea por árbol fluctuaron de 8.264 a $105.099 \mathrm{~kg}$ en fustes, de 0.329 a $21.442 \mathrm{~kg}$ en ramas y de 0.393 a $9.609 \mathrm{~kg}$ en follaje. A nivel de árbol $79.68 \%$ de la biomasa se encontró en fustes, $13.58 \%$ en ramas y 6.72 $\%$ en follaje.

Palabras clave: Ecuaciones alométricas, follaje, fuste, pesos fresco y seco, ramas

ABSTRACT. The aboveground biomass of Vochysia guatemalensis Donn. Smith., a species of ecological and economic importance in the south-southeast of Mexico, was estimated. Seven trees of four diameter categories $(10,15$, 20 and $25 \mathrm{~cm}$ ) were felled and then separated by structural component. Samples were obtained from each component, weighed fresh and then oven dried. To determine the dry weight and estimate the biomass per component and total tree in each category, allometric equations were fitted using nonlinear regression techniques. The values of aboveground biomass per tree ranged from 8,264 to $105,099 \mathrm{~kg}$ in stems, from 0.329 to $21.442 \mathrm{~kg}$ in branches and from 0.393 to $9.609 \mathrm{~kg}$ in foliage. At tree level $79.68 \%$ of the biomass was found in stems, $13.58 \%$ in branches and $6.72 \%$ in foliage.

Key words: Allometric equations, foliage, stem, fresh and dry weights, branches.

\section{INTRODUCCIÓN}

Vochysia guatemalensis Donn. Sm. es una especie heliófila de rápido crecimiento que se encuentra en bosques húmedos y muy húmedos tropicales (Holdridge 2000). Se distribuye desde México hasta Colombia; altitudinalmente, se encuentra entre los 0 y $1000 \mathrm{msnm}$, en precipitaciones promedio anual de 3000 a $5000 \mathrm{~mm}$ y temperatura prome- dio anual de 24 a $30^{\circ} \mathrm{C}$. Comúnmente crece en selvas secundarias en diferentes estadios de sucesión y en potreros, también se le encuentra en sistemas agroforestales y en plantaciones de restauración (Solis y Moya 2004). En México su distribución abarca desde el centro del estado de Veracruz hasta Tabasco, en la Chinantla y los Chimalapas de Oaxaca, y en la selva Lacandona de Chiapas (Pennington y Sarukhán 2005). Su rápido crecimiento la 
convierte en una especie con algunos de los mayores

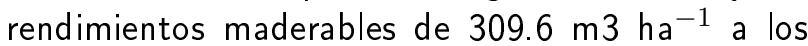
10 años (Redondo-Brenes 2007), con aportes de biomasa aérea por árbol de $112 \mathrm{~kg}$ en plantaciones del trópico húmedo Costarricense (Montero y Montagnini 2005).

El componente aéreo de los árboles es uno de los principales almacenes de biomasa. Lo que se traduce en la capacidad de los bosques para almacenar carbono y regular su balance a nivel global (Pardos 2010). La dinámica de la biomasa depende de la composición florística, edad y densidad de población en cada estrato de la comunidad vegetal (Laumonier et al. 2010). Hay dos métodos comúnmente usados para estimar la biomasa en comunidades forestales, los cuales son el método directo y el indirecto. El primero consiste en cortar la planta y determinar su biomasa pesando de forma directa cada componente; mientras que el segundo es un método menos intrusivo, estima la biomasa mediante ecuaciones o modelos alométricos basados en análisis de regresión con variables recolectadas en campo tales como diámetro a la altura del pecho (DAP), altura total, área basal y densidad especifica de la madera (Ketterings et al. 2001, Cutini et al. 2013). Este último método es extrapolable a condiciones de crecimiento y manejo similares de la misma especie (Picard et al. 2012). La biomasa aérea de $V$. guatemalensis se ha estimado por ambos métodos en plantaciones de Costa Rica (Montero y Montagnini 2005, Fonseca et al. 2009). Pero no existe suficiente información sobre $V$. guatemalensis, en selvas primarias o secundarias.

La Chinantla, es una región que comprende la tercera masa forestal más extensa del trópico húmedo mexicano, que se localiza en el norte del estado de Oaxaca (De Teresa et al. 2009). La cual ha sido sometida a cambios de uso del suelo para actividades agropecuarias, por lo que es común encontrar a $V$. guatemalensis en planicies, lomeríos moderados y en fragmentos de selva denudados (Pineda-Herrera et al. 2012). Con el propósito de contribuir al conocimiento de la productividad de estas selvas, el presente estudio tuvo el objetivo de ajustar ecuaciones alométricas para estimar la biomasa aérea total y su distribución en fuste, ramas y follaje de árboles de $V$. guatemalensis en una selva secundaria alta perennifolia.

\section{MATERIALES Y MÉTODOS}

El estudio se realizó en la localidad de San Martín Soyolapan del municipio de Comaltepec, cuenca del Río Papaloapan, en las estribaciones de la Sierra Norte de Oaxaca, ubicada a los $17^{\circ} 41^{\prime}$ 53.5" LN y $96^{\circ} 16^{\prime}$ 57.3" LO, a una altitud de 160 $\mathrm{m}$. La investigación se llevó a cabo en vegetación secundaria derivada de selva alta perennifolia con 20 años de sucesión en lo que anteriormente fue un terreno dedicado al cultivo de café, en un suelo arcilloso de hasta $30 \%$ de humedad en época de lluvias (Pineda-Herrera et al. 2012). El clima de la zona tiene temperaturas medias anuales de entre 16 y $25^{\circ} \mathrm{C}$, con precipitaciones medias anuales de 700 a 4000 mm (García 1988). Geológicamente se encuentra en un afloramiento de rocas ígneas extrusivas y calizas (INEGI 2015), con suelos Luvisoles (IUSS 2007). La vegetación predominante es la selva alta perennifolia con presencia de especies arbóreas como Andira galeotiana Standley, Brosimum alicastrum SW., Dialium guianense (Aub) Sandw., Terminalia amazonia (J. F. Gmel.) Exell y Vochysia guatemalensis Donn. Sm. (sensu Miranda y Hernández 1963).

Se seleccionaron siete árboles por sus características fenotípicas, su condición fitosanitaria y su ubicación con características topográficas, edáficas y climáticas similares. Se agruparon en cuatro categorías diamétricas en intervalos de 7.5 a $27.6 \mathrm{~cm}$ de diámetro a la altura del pecho (DAP). La distribución de los árboles por categorías diamétricas fue de dos individuos para 10,15 y $20 \mathrm{~cm}$ y un individuo para $25 \mathrm{~cm}$; el tamaño de muestra se determinó de acuerdo con Segura y Kanninen (2005). El muestreo se realizó en el mes de noviembre, época en la cual se presentan los mayores porcentajes de defoliación (Pineda-Herrera et al. 2012).

Se empleó el método directo para obtener los compartimentos de fuste, ramas y hojas (AcostaMireles et al. 2011, Ruiz-Aquino et al. 2014). Antes 
de derribar los árboles, se le midió el diámetro a la altura del pecho (DAP) con una cinta diamétrica Ben Meadows de $5 \mathrm{~m}$. Para luego medir la altura con una cinta métrica Sthil de $10 \mathrm{~m}$, cada árbol se dividió en fuste, ramas y hojas. Los fustes al igual que las ramas se cortaron en secciones de dos metros, el follaje se separó de las ramas y se empacó en bolsas de plástico de $0.5 \mathrm{~kg}$ (Picard et al. 2012). El fuste y las ramas se pesaron con una báscula Torrey Modelo CRS-500 con capacidad de 500 $\mathrm{kg} \pm 200 \mathrm{~g}$, para obtener el peso fresco en campo. El follaje y el peso fresco de las muestras se registró in situ para todos los compartimientos con una báscula de balanzón Oken Modelo E-5 con capacidad de $5 \mathrm{~kg} \pm 10 \mathrm{~g}$. Se obtuvieron cinco muestras por componente de cada árbol para todas las categorías diamétricas.

Las muestras de ramas y fustes se seccionaron de forma transversal en rodajas de 3 y $5 \mathrm{~cm}$ de grosor, respectivamente. Para luego depositarlas en bolsas de plástico para su traslado a los laboratorios generales del Colegio de Postgraduados campus Montecillo, donde se pusieron en bolsas de papel y se colocaron en una estufa de secado marca Felisa Modelo FE-293. Las muestras de ramas y de follaje se secaron a $75{ }^{\circ} \mathrm{C}$, mientras que las muestras de los fustes se secaron a $80^{\circ} \mathrm{C}$, hasta alcanzar peso constante. Con estos datos se estableció la relación entre peso fresco y peso seco, para estimar la proporción de biomasa por compartimientos, así como de la parte aérea total, empleando la siguiente ecuación (Picard et al. 2012):

$$
B=\frac{P F \operatorname{com} * P S c o m}{100}
$$

Donde: $B=$ biomasa del componente, PF$\mathrm{com}=$ peso fresco del componente, $\mathrm{PScom}=$ peso seco en kilogramos.

Con los datos de los pesos fresco y seco se obtuvieron valores de biomasa por compartimiento de cada árbol muestreado y se ajustaron ecuaciones alométricas mediante técnicas de regresión no lineal, empleando el método de mínimos cuadrados generalizados, en los cuales comprobaron los supuestos de normalidad y homogeneidad de varian- zas (Fonseca et al. 2009). El ajuste de los modelos potenciales se realizó con el módulo de análisis no lineal del paquete estadístico STATA. El modelo utilizado fue sigmoide, el cual se emplea con mayor frecuencia en la predicción del crecimiento biológico y en algunos estudios de biomasa arbórea (Lavigne et al. 1996, Hughes et al. 1999). Los modelos de Gompertz utilizados fueron los siguientes:

$y=\beta_{0} e^{\left(-e\left(\beta_{1}\left(D A P-\beta_{2}\right)\right)\right)}$

y $y=\beta_{0} e^{\left(-e\left(\beta_{1}\left(H-\beta_{2}\right)\right)\right)}$.

Donde: $\mathrm{y}=$ biomasa en $\mathrm{kg} ; \beta_{0}, \beta_{1}$ y $\beta_{2}=$ coeficientes de la regresión, DAP = diámetro a la altura del pecho en $\mathrm{cm}$ y $\mathrm{H}=$ altura en $\mathrm{m}$. Los indicadores de ajuste analizados fueron el coeficiente de determinación ajustado $\left(R^{2}\right)$, error estándar (EE), raíz del cuadrado medio del error (RCME) y significancia $(p<0.001)$ de los parámetros de cada ecuación (Álvarez-González et al. 2005, RojoMartínez et al. 2005).

\section{RESULTADOS Y DISCUSIÓN}

En las Figuras 1 y 2 se presentan las curvas de regresión ajustadas para cada uno de los componentes y para el total de los mismos respectivamente. En ambas figuras se presentan las ecuaciones alométricas y los estimadores de variación y confiabilidad estadística correspondientes. Las regresiones que involucraron al diámetro mostraron un buen nivel de ajuste $(p<0.0001)$, mientras que aquellas que se relacionan con la altura tuvieron bajos valores de $\mathrm{R}^{2}$ y los mayores valores de raíz de los cuadrados medios del error (RCME) (Figura 1, Tabla 1). Las curvas que relacionan la altura con la biomasa mostraron una redundancia de categorías de 10 a $15 \mathrm{~cm}$, por lo que no se apreció la conformación sigmoide característica del modelo Gompertz (Figura 2). Modelos sigmoides como el logístico en otros árboles de selva húmeda han mostrado ajustes con altos valores de $\mathrm{R}^{2}$ para el diámetro (0.99) y bajos para altura (0.70) (Hughes et al. 1999). EI DAP es una variable que se correlaciona con la biomasa de $V$. guatemalensis, por lo que 

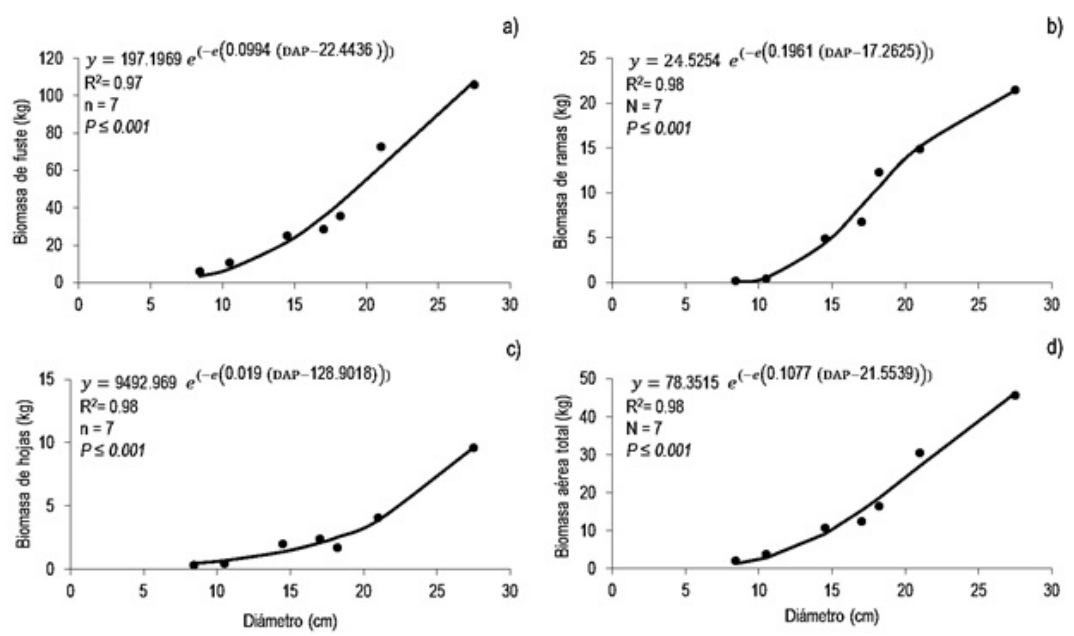

Figura 1. Curvas de regresión ajustada para estimar biomasa aérea por compartimientos: fustes (a), ramas (b), follaje (c) y total (d) de acuerdo al diámetro en árboles de Vochysia guatemalensis.

puede emplearse en inventarios dada su facilidad de medición en campo y extrapolarse a condiciones de crecimiento similares de forma no destructiva (Montero y Montagnini 2005). La poca predictibilidad del modelo con la altura de $V$. guatemalensis, se puede deber al hecho de que, en bosques no manejados, bajo condiciones de competencia por espacio y luz, el esfuerzo energético esta direccionado a crecer en altura para alcanzar el dosel, ponderándose la longitud antes que otras variables (Del-Amo y Nietode-Pascual 1981).

El porcentaje de la biomasa aérea en cada uno de los compartimientos de $\mathrm{V}$. guatemalensis, fue para el fuste de 77.33 a $91.96 \%$, en las ramas de 3.66 a $15.65 \%$ y en el follaje de 4.37 a $7.01 \%$. La distribución promedio fue de $79.68 \%$ en fuste, de $13.58 \%$ en ramas y de $6.72 \%$ en follaje (Tabla 2). Al respecto Montero y Montagnini (2005) encontraron datos promedio de biomasa en plantaciones de $V$. guatemalensis de $97.2 \mathrm{~kg}$ de fuste, $12.1 \mathrm{~kg}$ de ramas, $4.8 \mathrm{~kg}$ de hojas y $112.7 \mathrm{~kg}$ para el árbol total. Mientras que Goodman et al. (2014) mencionan que la biomasa está determinada por las dimensiones y la arquitectura de la copa, así como por la calidad de sitio y las características del rodal, entre las que destaca el espacio de crecimiento (Foli et al. 2003).
La distribución del peso seco en los diferentes componentes estructurales osciló de 8.26 a 105.09 $\mathrm{kg}$ en fuste; de 0.32 a $21.44 \mathrm{~kg}$ en ramas y de 0.39 a $9.60 \mathrm{~kg}$ en follaje. En todas las categorías diamétricas, la mayor proporción de biomasa aérea se encontró en el fuste y la menor en el follaje. Los valores de biomasa total a nivel de árbol oscilaron de 8.98 a $136.99 \mathrm{~kg}$ (Tabla 2). Valores que son menores a los reportados por Segura y Kanninen (2005) para el fuste $(65 \%)$ en selvas con Vochysia ferruginea Mart. El estado sucesional en la muestra de selva estudiada tiene implicaciones en los patrones de biomasa encontrada. Al respecto Jordan (1985) menciona que disturbios de moderada intensidad, pequeña extensión y corta duración permiten reestablecer la productividad y la acumulación de biomasa por contribución de los árboles circundantes al claro en la selva, permitiendo que especies pioneras y clímax repueblen el lugar. El perfil de $V$. guatemalensis como especie pionera de rápido crecimiento, la convierten en una especie recuperadora de biomasa y por lo tanto de la productividad en esta selva (Solis y Moya 2004).

El patrón de distribución de la biomasa de $V$. guatemalensis, con respecto al peso seco total, indica que esta especie tiene su mayor potencial para acumular biomasa en fuste en categorías 
Tabla 1. Valores de los parámetros y estadísticos de regresión del modelo ajustado para estimar la biomasa aérea de Vochysia guatemalensis de acuerdo al diámetro y la altura.

\begin{tabular}{lcrrrrrr}
\hline Componente & Parámetro & Valor & Error estándar & $\mathrm{RCME}$ & Valor $\mathrm{t}$ & $\mathrm{P}>|\mathrm{t}|$ & $\mathrm{R}^{2}$ ajustado \\
\hline \multirow{2}{*}{ fuste } & $\beta_{0}$ & 197.1969 & 105.3622 & & & & \\
& $\beta_{1}$ & 0.0994 & 0.0494 & 7.6719 & 1.87 & 0.135 & \\
& $\beta_{2}$ & 22.4436 & 5.9222 & & 3.79 & 0.019 & 0.97 \\
ramas & $\beta_{0}$ & 24.5254 & 3.0997 & & 7.91 & 0.001 & \\
& $\beta_{1}$ & 0.1961 & 0.0524 & 1.2351 & 3.74 & 0.020 & 0.98 \\
& $\beta_{2}$ & 17.2625 & 0.8335 & & 20.71 & 0.000 & \\
hojas & $\beta_{0}$ & 9492.969 & 17.1850 & & 0.06 & 0.959 & \\
& $\beta_{1}$ & 0.019 & 0.0451 & 0.5602 & 0.42 & 0.695 & 0.98 \\
árbol & $\beta_{2}$ & 128.9018 & 378.354 & & 0.34 & 0.750 & \\
Altura & $\beta_{0}$ & 78.3515 & 28.7943 & & 2.72 & 0.053 & \\
fuste & $\beta_{1}$ & 0.1077 & 0.0403 & 2.6203 & 2.67 & 0.056 & 0.98 \\
& $\beta_{2}$ & 21.5539 & 3.8575 & & 5.59 & 0.005 & \\
ramas & $\beta_{0}$ & 60.5453 & 17.3583 & & 3.49 & 0.025 & \\
& $\beta_{1}$ & 2.0754 & 20.4083 & 31.9446 & 0.10 & 0.924 & 0.63 \\
& $\beta_{2}$ & 12.3492 & 1.1209 & & 11.02 & 0.000 & \\
hojas & $\beta_{0}$ & 13.8392 & 3.2898 & & 4.21 & 0.014 & \\
& $\beta_{1}$ & 2.0888 & 17.5567 & 5.6301 & 0.12 & 0.911 & 0.75 \\
árbol & $\beta_{2}$ & 12.5023 & 2.1415 & & 5.84 & 0.004 & \\
& $\beta_{0}$ & 4.4457 & 1.8077 & & 2.46 & 0.070 & \\
& $\beta_{1}$ & 2.0083 & 27.5396 & 3.1777 & 0.07 & 0.945 & 0.42 \\
\hline & $\beta_{2}$ & 12.3847 & 2.0147 & & 6.15 & 0.004 & \\
& $\beta_{0}$ & 26.2759 & 7.4003 & & 3.55 & 0.024 & \\
& $\beta_{1}$ & 2.0673 & 19.9678 & 13.4432 & 0.10 & 0.923 & 0.64
\end{tabular}

Fecha 1: $32 \mathrm{~d}$, Fecha 2: $62 \mathrm{~d}$, Fecha 3: $93 \mathrm{~d}$
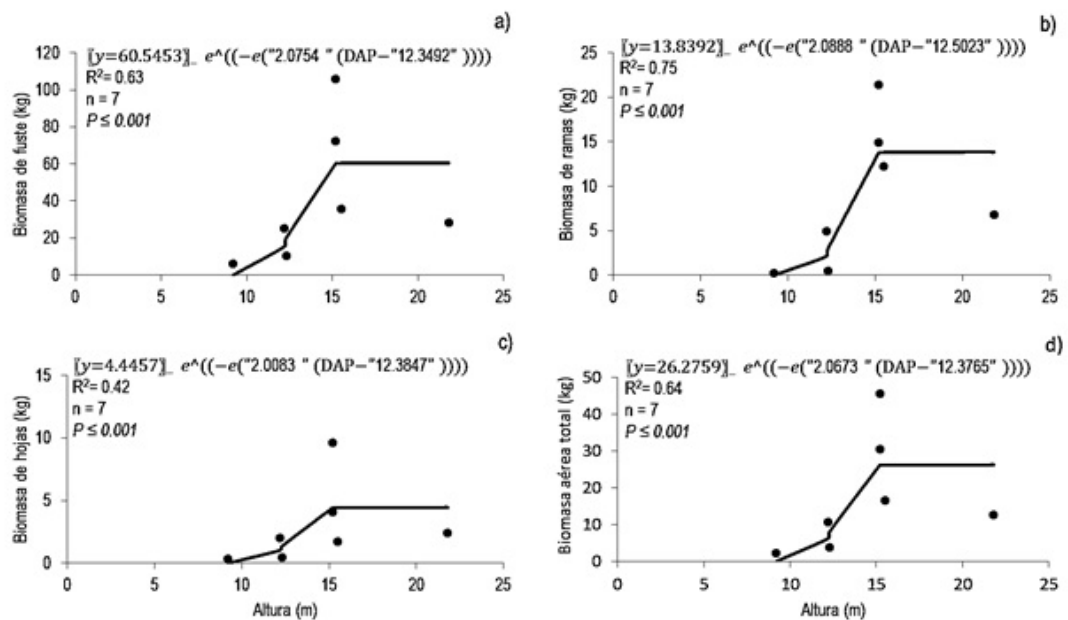

Figura 2. Curvas de regresión ajustada para estimar biomasa aérea por compartimientos: fustes (a), ramas (b), follaje (c) y total (d) de acuerdo a la altura en árboles de Vochysia guatemalensis.

menores y disminuye conforme crece en diámetro, favoreciendo a las ramas y hojas. Las ecuaciones alométricas generadas mediante regresión no lineal para el diámetro mostraron un buen nivel de ajuste, determinado por sus parámetros estadísticos, por lo que el modelo Gompertz es confiable para realizar una buena predicción de la biomasa aérea. 
Tabla 2. Peso seco $(\mathrm{kg})$ y distribución de biomasa aérea (\%) de cada uno de los componentes estructurales de las diferentes categorías diamétricas de $V$. guatemalensis.

\begin{tabular}{lrrrrrrrr}
\hline $\mathrm{CD}$ & \multicolumn{3}{c}{ Peso seco $(\mathrm{kg})$} & & \multicolumn{3}{c}{ Distribución de biomasa $(\%)$} \\
\cline { 2 - 4 }$(\mathrm{cm})$ & fuste & ramas & follaje & árbol & & fuste & ramas & follaje \\
\hline 10 & 8.2644 & 0.3291 & 0.3932 & 8.9868 & & 91.961 & 3.6626 & 4.3761 \\
15 & 26.7582 & 5.8504 & 2.2006 & 34.809 & & 76.871 & 16.8071 & 6.3218 \\
20 & 54.0994 & 13.5872 & 6.8462 & 74.533 & & 72.585 & 18.2298 & 9.1855 \\
25 & 105.0994 & 21.4427 & 9.6097 & 136.99 & & 77.333 & 15.6522 & 7.0146 \\
\hline Promedio & 40.5980 & 8.7109 & 2.9416 & 52.2505 & & 79.6874 & 13.5879 & 6.7245 \\
\hline CD: Categoría diamétrica. & & & & & & &
\end{tabular}

\section{AGRADECIMIENTOS}

A la fundación FORD, al comisariado comunal de Santiago Comaltepec, a las autoridades y los habitantes de la agencia municipal de San Martín Soyolapan por su apoyo. A los revisores asignados por sus sugerencias y comentarios al manuscrito.

\section{LITERATURA CITADA}

Acosta-Mireles M, Carrillo-Anzures F, Gómez Villegas R (2011) Estimación de biomasa y carbono en dos especies de bosque mesófilo de montaña. Revista Mexicana de Ciencias Agrícolas 2: 529-543.

Álvarez-González JG, Balboa-Murias MA, Merino A, Rodríguez-Soalleiro R (2005) Estimación de la biomasa arbórea de Eucalyptus globulus y Pinus pinaster en Galicia. Recursos Rurais 1: 21-30

Cutini A, Chianucci F, Manetti MC (2013) Allometric relationships for volume and biomass for stone pine (Pinus pinea L.) in Italian coastal stands. Forest-Biogeosciences and Forestry 6: 331-335.

Del Amo RS, Nieto de Pascual C (1981) Applications of models and mathematical equations to evaluate growth rates and age determination of tropical trees. In: Bormann FH, Berlyn G (ed). Age and growth rate of tropical trees. Yale, USA. pp: 128-133.

De Teresa AP, Hernández G, Legarreta P, Orozco M (2009) Quia-na. La selva chinanteca y sus pobladores. UAM-I-Juan Pablos editores, México, DF. 113p.

Foli EG, Alder D, Miller HG, Swaine MD (2003) Modelling growing space requirements for some tropical forest tree species. Forest Ecology and Management 173: 79-88.

Fonseca GW, Alice F, Rey JM (2009) Modelos para estimar la biomasa de especies nativas en plantaciones y bosques secundarios en la zona Caribe de Costa Rica. Bosque 30: 36-47.

García E (1988) Modificaciones al sistema climático de Köppen adaptado para México. Instituto de Geografía, UNAM. México. 90p.

Goodman RC, Phillips O, Baker TR (2014) The importance of crown dimensions to improve tropical tree biomass estimates. Ecological Applications 24: 680-698.

Holdridge L (2000) El diagrama de las zonas de vida. Ecología basada en zonas de vida. 5ta. Reimpresión. Instituto Interamericano de Ciencias Agrícolas. San José, Costa Rica. 216p.

Hughes RF, Kauffman JB, Jaramillo VJ (1999) Biomass, carbon, and nutrient dynamics of secondary forests in a humid tropical region of Mexico. Ecology 80: 1892-1907.

INEGI (2015) Instituto Nacional de Estadística y Geografía. Prontuario de información geográfica municipal de los Estados Unidos Mexicanos. http://www.inegi.org.mx/sistemas/mexicocifras/datos-geograficos/ 12/12038.pdf?. Fecha de consulta 18 de octubre de 2015. 
IUSS (2007) Base Referencial Mundial del Recurso Suelo. Primera actualización. Informes sobre Recursos Mundiales de Suelos No. 103. FAO. Roma. 201p.

Jordan CF (1985) Nutrient cycling in tropical forest ecosystems. Principles and Their Application in Management and Conservation. John Wiley \& Sons. Chichester, USA. 179p.

Ketterings QM, Coe R, van Noordwijk M, Palm CA (2001) Reducing uncertainty in the use of allometric biomass equations for predicting above-ground tree biomass in mixed secondary forests. Forest Ecology and management 146: 199-209.

Laumonier Y, Edin A, Kanninen M, Munandar AW (2010) Landscape-scale variation in the structure and biomass of the hill dipterocarp forest of Sumatra: Implications for carbon stock assessments. Forest Ecology and Management 259: 505-513.

Lavigne MB, Luther JE, Franklin SE, Hunt Jr ER (1996) Comparing branch biomass prediction equations for Abies baisamea. Canadian Journal of Forest Research 26: 611-616.

Miranda F, Hernández E (1963) Los tipos de vegetación de México. Boletín de la Sociedad Botánica de México 28: 29-179.

Montero M, Montagnini F (2005) Modelos alométricos para la estimación de biomasa de diez especies nativas en plantaciones en la región Atlántica de Costa Rica. Recursos Naturales y Ambiente 45: 118-125.

Pardos JA (2010) Los ecosistemas forestales y el secuestro de carbono ante el calentamiento global. Instituto Nacional de Investigación y Tecnología Agraria y Alimentaria (INIA). Ministerio de Ciencia e Innovación. Madrid, España. 253p.

Pennington TD, Sarukhán J (2005). Árboles tropicales de México: Manual para la identificación de las principales especies. Texto Científico Universitario. Tercera edición. Fondo de Cultura Económica. UNAM. México, DF. 523p.

Picard N, Saint-André L, Henry M (2012) Manual de construcción de ecuaciones alométricas para estimar el volumen y la biomasa de los árboles: Del trabajo de campo a la predicción. S.I. Centre de Coopération Inernationale en Recherche Agronomique pour le Développement (CIRAD) y Las Naciones Unidas para la Alimentación y la Agricultura (FAO). Roma, Italia. 213p.

Pineda-Herrera E, Valdez JI, López MA (2012) Fenología de dos especies arbóreas en una selva alta perennifolia del Norte de Oaxaca. Botanical Sciences 90: 185-193.

Redondo-Brenes A (2007) Growth, carbon sequestration, and management of native tree plantations in humid regions of Costa Rica. New Forests 34: 253-268.

Rojo-Martínez GE, Jasso-Mata J, Vargas-Hernández JJ, Palma-López DJ, Velázquez-Martínez A (2005) Biomasa aérea en plantaciones comerciales de hule (Hevea brasiliensis Müll. Arg.) en el estado de Oaxaca, México. Agrociencia 39: 449-456.

Ruiz-Aquino F, Valdez-Hernández JI, Manzano-Méndez F, Rodríguez-Ortiz G, Romero-Manzanares A, FuentesLópez ME (2014) Ecuaciones de biomasa aérea para Quercus laurina y Q. crassifolia en Oaxaca. Madera y Bosques 20: 33-48.

Segura M, Kanninen M (2005) Allometric models for tree volume and total aboveground biomass in a tropical humid forest in Costa Rica1. Biotropica 37: 2-8.

Solis M, Moya R (2004) Vochysia guatemalensis en Costa Rica. COSEFORMA-GTZ. San José, Costa Rica. $100 \mathrm{p}$. 
Manzano-Méndez et al.

Ecosistemas

Pecursos

Cigropecuarios
Biomasa aérea de Vochysia guatemalensis

Ecosist. Recur. Agropec. 4(11):395-402,2017

West PW (2006) Growing plantation forests. Springer-Verlag. Berlin, Germany. 304p. 